\title{
ANALYSIS OF VIBRATION DETECTION USING ACTIVE CONTROLLER IN THE SMART CANTILEVER \\ COMPOSITE BEAM WITH LQR AND
}

\author{
FUZZY TECHNIQUES \\ F. FREDRICK GNANARAJ ${ }^{1} \&$ K. R. VIJAYA KUMAR ${ }^{2}$ \\ ${ }^{I}$ Research Scholar, Department of Mechanical Engineering, St. Peter's Institute of \\ Higher Education and Research, Chennai, Tamil Nadu, India \\ ${ }^{2}$ Professor, Department of Mechanical Engineering, Dr. M. G. R. Educational and
}

Research Institute, Chennai, Tamil Nadu, India

\begin{abstract}
Vibration is the major problem in light weight composite structures. Active vibration methods are effective to control the vibration. In this work, vibration control using active controller for an E-Glass/Epoxy Cyanate laminated composite beam, which uses patches of piezoelectric ceramic patches as smart sensors and actuators were tested and analyzed. The combination of Linear Quadratic Regulator (LQR) with Kalman filter and Fuzzy technique gives greater Region Of Convergence (ROC). The control is based on the proximity output and the previous output. This combination gives best optimization in the output, hence the settling time is good, and the system gives fast and stable output response. The entire Vibration Reduction System (VRS) consists of a Data Acquisition module, composite beam with Piezo electric ceramic sensor and actuator. This VRS setup is simulated using Matlab/Simulink and the output analysis shows that, this system reduces the vibration efficiently compared to the previous methods.
\end{abstract}

KEYWORDS: Linear Quadratic Regulator (LQR), Kalman Filter, Vibration Reduction System (VRS) \& Fuzzy Logic Controller FLC)

Received: Jul 12, 2018; Accepted: Aug 06, 2018; Published: Aug 24, 2018; Paper Id.: IJMPERDOCT20189

\section{INTRODUCTION}

Recently, composites, which are light weight, are used in many modern industries, especially for aerospace and spacecraft applications. Due to vibration, expected output could not be obtained, which is a major challenge in composite structures, hence numerous research work is carried out to detect and also to control the vibration. The two methods to control vibration are passive and active methods. In passive vibration control, structural modifications are used to control vibrations (Maed J D, 1999). Dynamic absorbers, dampers or neutralizers are used for the passive vibration control (Eissa, 2000). To increase the stiffness and reduce low frequency vibration of a system, active vibration is preferable. In active vibration control, the vibration is reduced by using sensors, controllers and actuators (Sen M. Kuo, et al., 1999), (Fuller, et al., 1995), (Leventhall, et al., 1998) (Premount, 2001). The sensors measure the vibration and convert it into a measurable signal, and this information is given as input to the controller which makes decisions based on the control algorithm and gives control command to the actuating unit, which has a mechanism to control the system (Labane, 2014). The 
comparison between the properties of active and passive vibration control methodology shows that active vibration control is better compared to passive vibration control (Fuller 1995). Passive control is considered as open loop system, while active control is considered as closed loop system. Active control is used for low frequency vibration control, while high frequency vibration can be eliminated by using passive control.

The desirable characteristics of piezoelectric sensors and actuators are, easily bonded, easily manufactured in the desired shape, light weight, high actuating force, high stiffness, good vibration attenuation, low power consumption and cost effective (Premount, 2001). Fuzzy Logic gives good optimization for complex control analysis, and this controller performance is better compared to other controllers for closed loop damping analysis (Anshul, 2013). It reduces the unwanted vibration using 'if then else' rules. Fuzzy logic gives good resolution and good accuracy in the output, which is very useful in real time tuning application (He Rhonbo, 2013). Active vibration control strategy has been implemented in various applications like helicopter modules.

The controller plays a key role to control vibration, which is an interface between the system and actuator. In this work, the efficiency of the controller is compared between LQR with Kalman filter and LQR, Kalman filter with Fuzzy controller. Generally, feedback control helps in the control activity verification. The feedback controller takes the output as one of the input and compares it with the output response, which is given to the system to control the parameters. Feed forward control is more advantage compared to the feedback controller, because it works proactively instead of reactively (Leventhall, 1998). Feed forward control initially checks the external excitation changes and corresponding pre-planned activities is taken in the system.

\section{LITERATURE SURVEY}

Active control balances the damping losses, and hence maximizes the isolation. A four-fold model was experimented, and hence proved that, RMS (Root Mean Square) force which was transmitted is reduced and also the fundamental frequency is reduced, thus providing a wide frequency range isolation (Jean-Paul Henderson, 2018). In this research work, both the vibration control, active and passive, performance compared and were analyzed, and it was observed that though passive vibration system is having small percentage overshoot compared to active vibration control system, settling time of passive is poor compared to active controller (Faried Hasbullah, 2010).

Vibration control is obtained in less time using LQR control techniques in cantilever composite beam (Rahman, et al. 2018). Closed loop system with fast transient response and zero steady state error is preferred for many applications, and therefore PID controllers are preferred for many applications, because it satisfies both the properties at the same time. The major drawback of PD and PID controller is that, they will not consider huge uncertainties and also the vibrations of lateral-torsional are not considered. Fuzzy logic is an efficient way to compensate the uncertainties (Satyam Paul, 2018). Response and settling time is better in cantilever beam and, using fuzzy controller gives better control in vibration compared to PID controller and Linear Quadratic Regulator (LQR) controller (Tahseen, 2014). Fuzzy Logic controller is very robust with respect to change in input commands and noise vibration, and also gives accurate output. LQR is optimal controller which minimizes the cost function. LQR and Fuzzy guarantees a stable output and damping is minimized extraordinarily compared to other controllers (Ali Ajami, 2009). Thus, in this work, combination of LQR with Kalman filter and Fuzzy gives good dynamic response than other controllers. 


\section{METHODOLOGY}

In this research, the main objective is to reduce vibration using active vibration methodology. The composite beam used is E-glass/Epoxy, which is fixed in cantilevered position. For modal, a Finite Element Analysis (FEA) of the laminated composite beam is used for modal frequencies. To obtain active vibration and suppression in composite beam, patches of two PZT lead zirconatetitanate are attached to the beam on both sides, one is used as a sensor and other is used as an actuator. The VRS consists of composite beam, which is attached with PZT actuator and sensor, digital data acquisition system, voltage amplifier and real-time control system.

\subsection{Modelling of Cantilever Beam}

The Proportional-Integral-Derivative (PID) is a feedback controller methodology, which determines the error (e) between desired value and measured value, and this error value is used to regulate the input of the system to attain the defined set value. LQR is a control methodology, which improves the performance of vibration control. The main objective of designing a LQR is to formulate feedback controller A with gain matrix, such that it minimizes the objective function B, and hence achieves a stable system.

The continuous linear time system is given by the equation (1)

$$
\dot{x}=C x+D u
$$

The cost functions are defined with weight matrices of $\mathrm{M}$ and $\mathrm{N}$ in equation (2)

$$
B=\int_{0}^{\infty}\left(x^{t} M x+u^{t} N u\right) d t
$$

The matrices $\mathrm{M}$ and $\mathrm{N}$ is made diagonal matrix, such that $\mathrm{M}$ and $\mathrm{N}$ will be positive definite symmetry matrix.

The equation (3) is a feedback control rule, which reduces the cost:

$$
u=-A x
$$

A is given by, $A=P^{-1} D^{T} R$

The value of A can be determined from equation (4), and R value can be determined by solving Riccati equation (5)

$$
C^{T} R+R C-R D N^{-1} D R+N=0
$$

Compared to other controllers, LQR controller is more advantageous, because it is robust for longitude and lateral flight dynamics of an aircraft control system, and hence achieves great dynamic performance and also good stability in aircraft networks [7].

\subsection{LQR with Kalman Filter}

In this system, LQR with Kalman filter is used for the vibration control of composite material. In this circuit, to optimize the output of the system, LQR gain matrix and feed forward gain blocks are used. Compared to feedback control, feed forward has the advantage of pre-defined way of output response, without expecting for the output feedback. In circuit simulation, step input is used as desired input to obtain measured and predicted output. The input to the Kalman filter is the output of sensor noise and actuator. The state of the system is approximated using measured output and Kalman filter. 
Actual input and demanded input is given to the actuator. From this system, the estimated output is obtained. Figure 1 illustrates the Matlab/Simulink circuit diagram of VRS. Figure 2 shows the vibration control graph, which proves that vibration, reduces gradually. Figure 3 is the optimization graph.

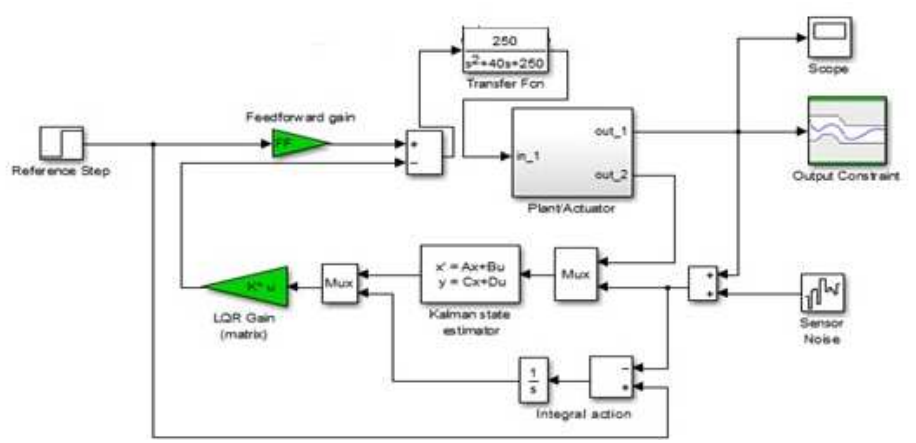

Figure 1: LQR Controller for the Vibration Control of Composite Material

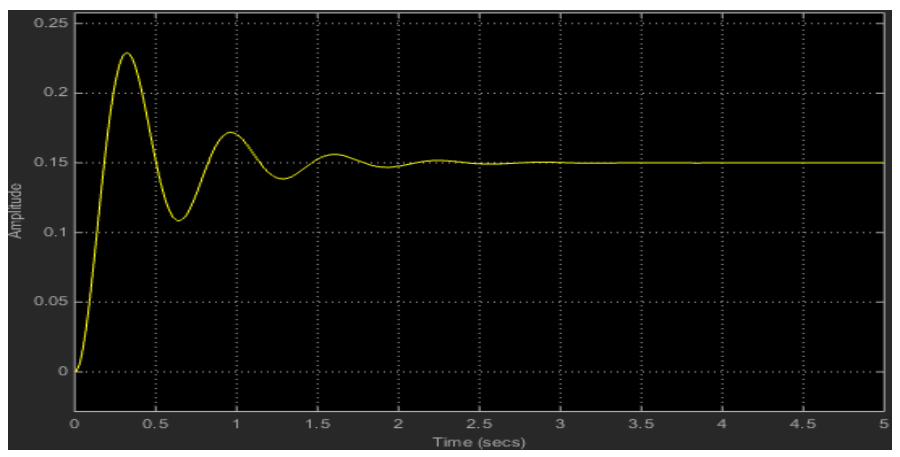

Figure 2: Vibration Control with LQR and Kalman Filter

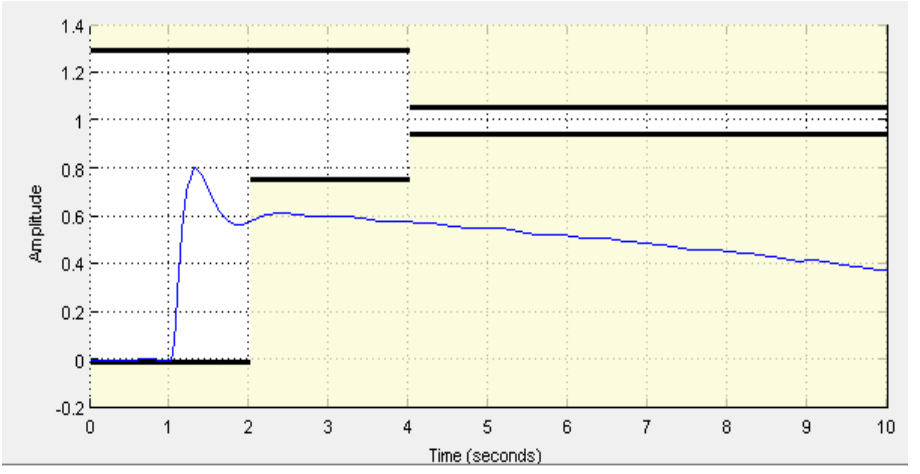

Figure 3: Optimization of LQR with Kalman Filter

\begin{tabular}{|c|c|c|c|}
\hline Iteration & Fecount & $\begin{array}{l}\text { Output Constraint (Uppes) } \\
\qquad k=0)\end{array}$ & $\begin{array}{l}\text { Output Constraint (Lower) } \\
\qquad(p=0)\end{array}$ \\
\hline 0 & 39 & .02323 & -0.6083 \\
\hline 1 & 67 & 0.4580 & .00955 \\
\hline 2 & 101 & 0.0195 & .0 .0141 \\
\hline 3 & 135 & 0.0013 & $307656-04$ \\
\hline 4 & 169 & $342940-05$ & $-1.69276-07$ \\
\hline
\end{tabular}

Figure 4: Iteration for Optimization Process 
Figure 4 shows the iteration for optimization process. Thus the optimization is obtained after four iterations.

\subsection{LQR with Kalman Filter and Fuzzy Controller}

In this work, Fuzzy controller is included in addition to LQR, with Kalman filter to reduce the vibration.

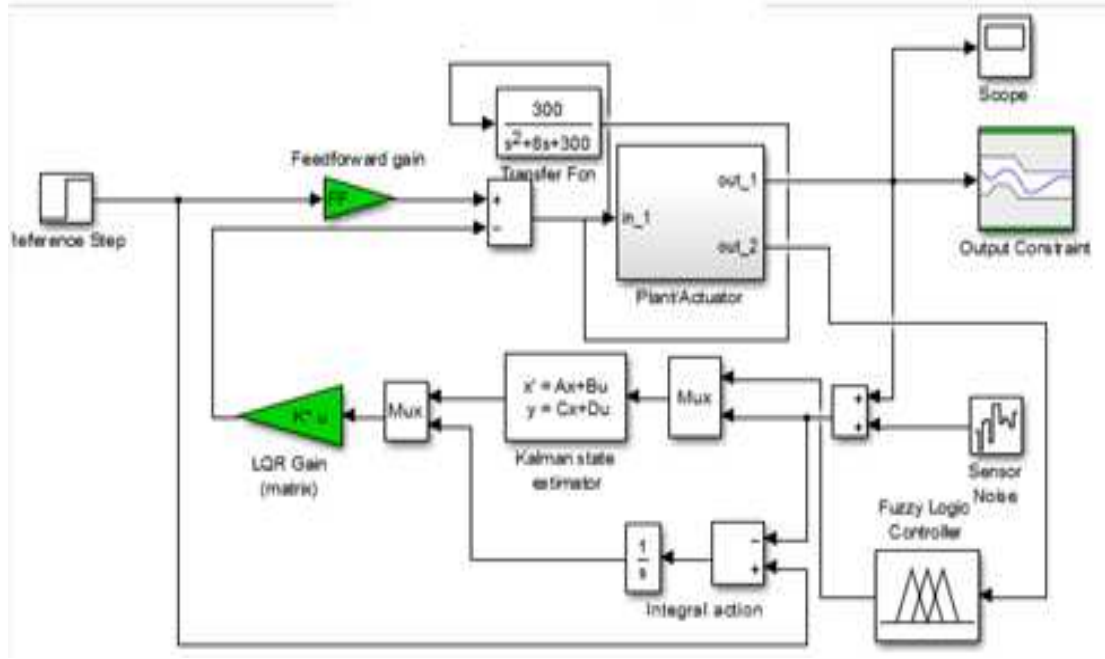

Figure 5: LQR with Kalman Filter and Fuzzy Controller

Figure 5 shows the Matlab Simulink circuit simulation with LQR, Kalman filter and Fuzzy controller. In this simulation circuit, Fuzzy Logic Controller is used before the Kalmanfilter, followed by LQR Gain Matrix. The inputs to FIS (Fuzzy Inference System) are previous state and present state of the system. By using Mamdani model in the fuzzy, the predicted output is generated. Figure 6 shows the FIS initialization block and Figure 7, Figure 8 and Figure 9 show the membership function of previous state input, membership function of present state input and membership function of predicted output, respectively.

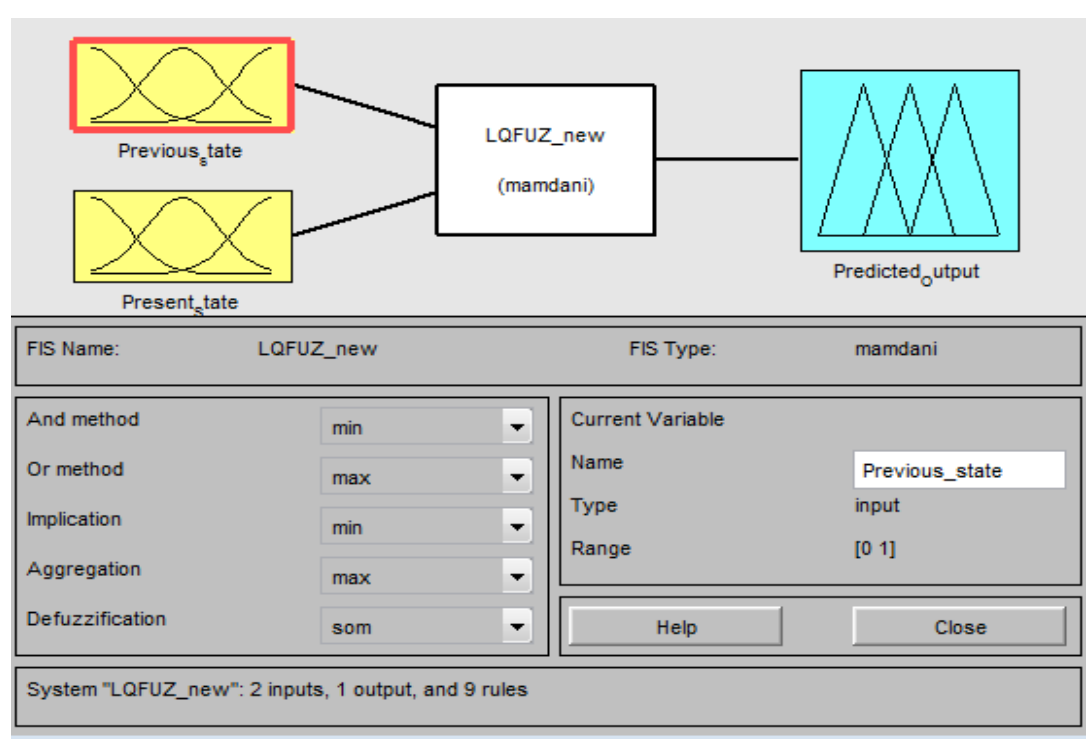

Figure 6: FIS Initialization Block 


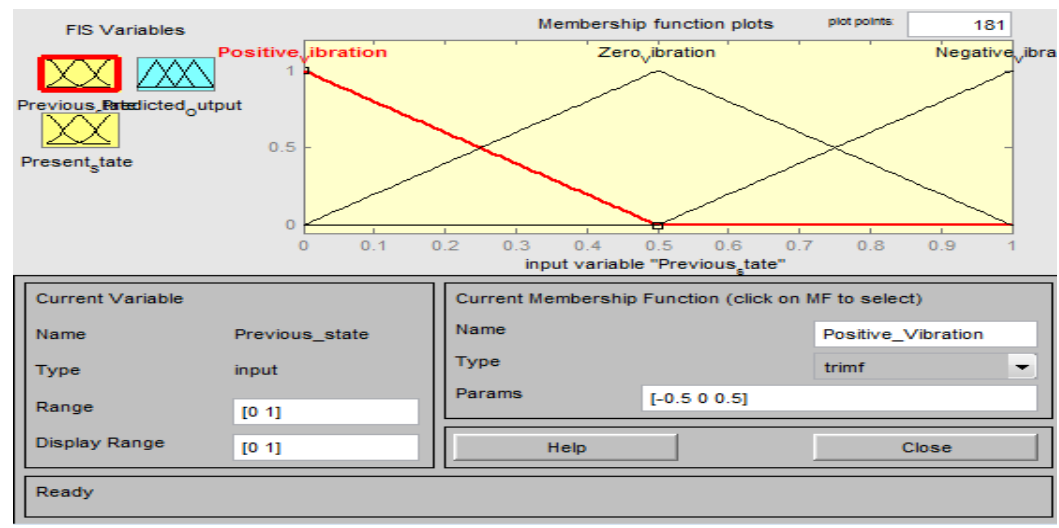

Figure 7: Fuzzy Membership Function of Previous State Block Input

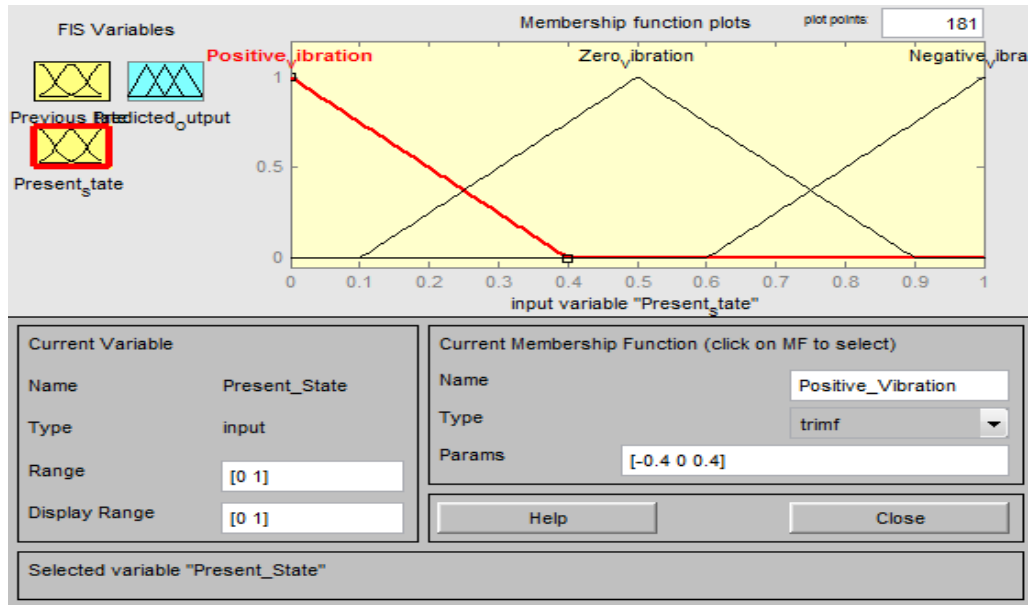

Figure 8: Fuzzy Membership Function of Present State Block Input

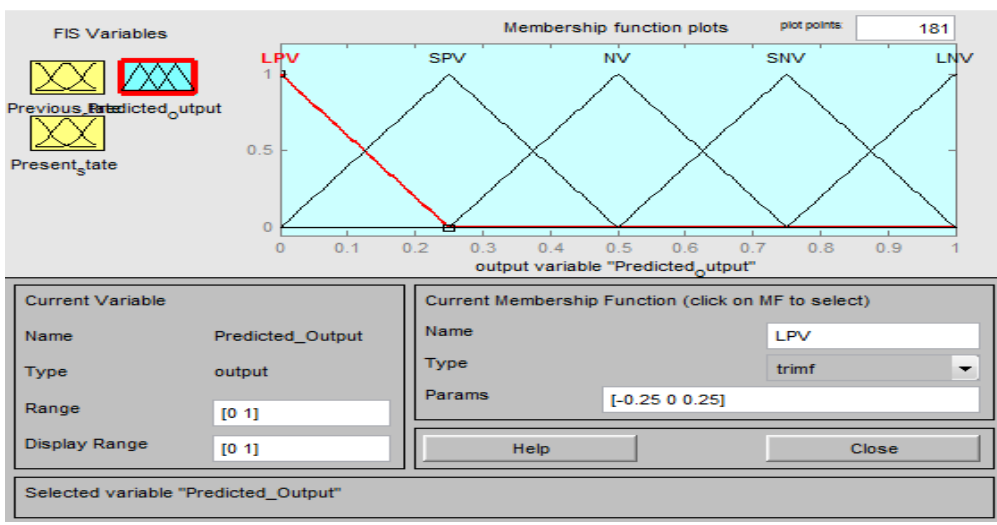

Figure 9: Fuzzy Membership Function of Predicted Output Block

\section{RESULTS AND DISCUSSIONS}

Vibrations are classified into three types namely Positive, Negative and Zero vibrations, which is given as input to the system. The outputs of the system are classified into Large Positive Vibration, Small Positive Vibration, Negative Vibration, Small Negative Vibration and Large Negative Vibrations. Table 1 shows the expected output for present input and previous output. These are framed as fuzzy rules, which can be viewed in Figure 10. 
Table 1: Fuzzy Control

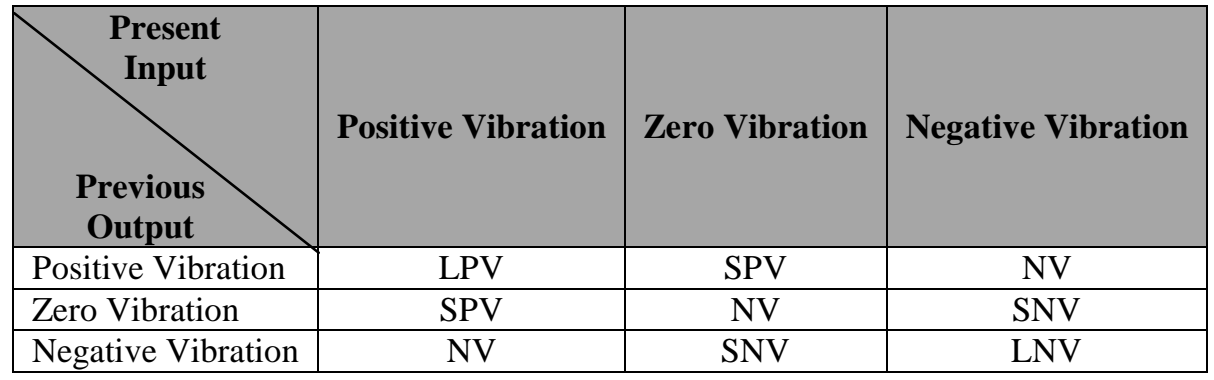

LPV - Large Positive Vibration

SPV - Small Positive Vibration

NV - Negative Vibration

SNV - Small Negative Vibration

LNV - Large Negative Vibration

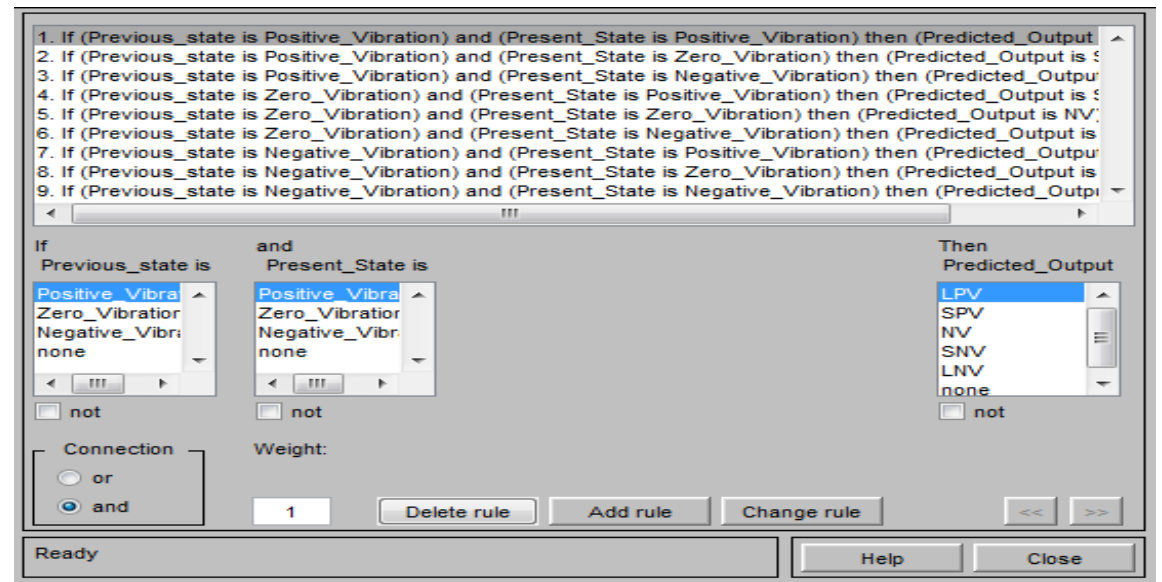

Figure 10: Rule Editor in Fuzzy Logic

The fuzzy rules are,

- If (Previous_state is positive vibration) and (Present_state is Positive_Vibration) then (Predicted_out is Large Positive Vibration)

- If (Previous_state is positive vibration) and (Present_state is Zero_Vibration) then (Predicted_out is Small Positive Vibration).

- If (Previous_state is positive vibration) and (Present_state is Negative_Vibration) then (Predicted_out is Large Negative Vibration).

- If (Previous_state is Zero vibration) and (Present_state is Positive_Vibration) then (Predicted_out is Small Positive Vibration).

- If (Previous_state is Zero vibration) and (Present_state is Zero_Vibration) then (Predicted_out is No Vibration).

- If (Previous_state is Zero vibration) and (Present_state is Negative_Vibration) then (Predicted_out is Small Negative Vibration).

- If (Previous_state is Negative vibration) and (Present_state is Positive_Vibration) then (Predicted_out is Large No 
Vibration).

- If (Previous_state is Negative vibration) and (Present_state is Zero_Vibration) then (Predicted_out is Small Negative Vibration).

- If (Previous_state is Negative vibration) and (Present_state is Negative_Vibration) then (Predicted_out is Large Negative Vibration).

The predicted output is observed from the graph. It is clear that, LQR with Fuzzy Logic combination can give optimized output as compared to LQR with Kalman filter. Figure 11 shows the predicted output of fuzzy. Thus, the final output of the system using LQR with Kalman filter and Fuzzy Controller is shown in Figure 12.

The comparison between the performance of LQR with Kalman filter and LQR with Kalman and Fuzzy controller is tabulated in Table 2. From Table 2, it is clear that using Fuzzy technique combined with LQR and Kalman Filter gives fast settling time and the amplitude of vibration is reduced. Fuzzy is used to limit the number of iterations, and also it improves the stiffness factor. Thus, a non-linear problem is solved, as if the problem is linear, using fuzzy controller. Since previous output and present inputs were combined to frame if then else rules, the displacements could be predicted, therefore, the vibration settling time is earlier.

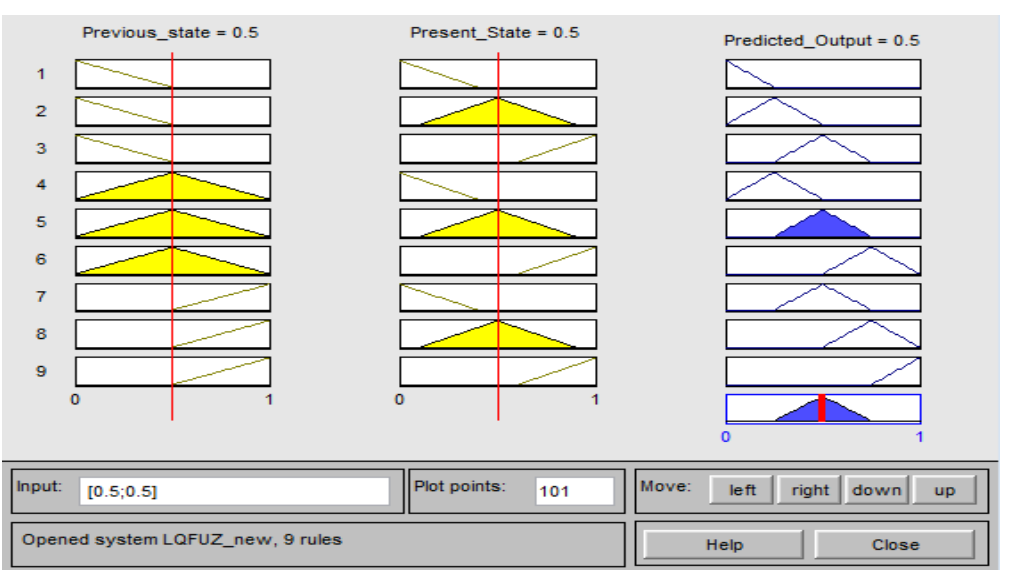

Figure 11: Rule Viewer

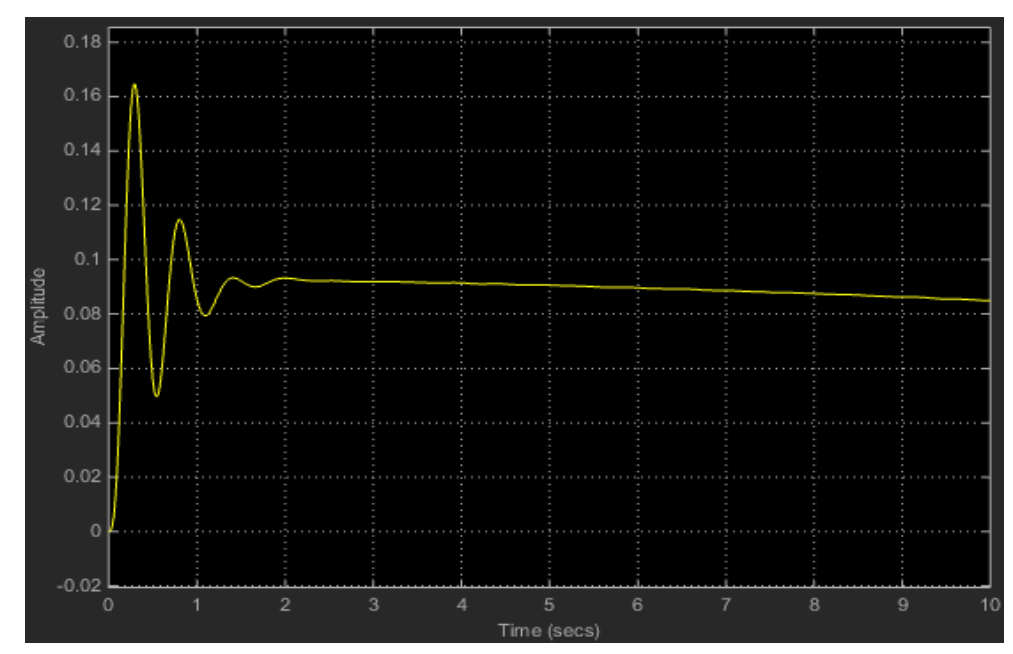

Figure 12: Vibration Control Output Using LQR with Kalman Filter and Fuzzy Controller 
Table 2: Performance Comparison of LQR, Kalman Filter and LQR with Kalman Filter, Fuzzy Controller

\begin{tabular}{|c|l|c|c|}
\hline Si. No. & \multicolumn{1}{|c|}{ Controller } & Time Period & Amplitude \\
\hline 1 & LQR With Kalman Filter & $2.2 \mathrm{sec}$ & $0.23 \mathrm{~mm}$ \\
\hline 2 & LQR with Kalman and Fuzzy Controller & $2 \mathrm{sec}$ & $0.1 \mathrm{~mm}$ \\
\hline
\end{tabular}

\section{CONCLUSIONS}

In this work, Active vibration control is obtained in the composite beam with PZT sensors, controllers and actuators. The combination of LQR and Kalman Filter with fuzzy controller is designed to obtain the control of vibration. The time period and amplitude is compared in both the methods. LQR, Kalman filter with Fuzzy gives good optimization compared to LQR with Kalman filter.

\section{REFERENCES}

1. Ali Ajami, Nasar Taheri and Mostafa Younesi. (2009). A Novel Hybrid Fuzzy/LQR Damping Oscillations Controller using STATCOM, International Conference on Computer and Electrical Engineering, Pg. 348-352.

2. Anshul Sharma and C. K. Susheel. (2013). Fuzzy based Active Vibration Controller, Applied Mechanics and Materials, Vol. 367, PP 357-362.

3. Eissa M. (2000). Vibration and chaos control in I. C engines subject to harmonic torque via non-linear absorbers, ISMV, Second International Symposium on Mechanical Vibrations. Islamabad, Pakistan, 2000.

4. Faried Hasbullah \& Waleed F. Faris. (2010). A Comparative Analysis of Fuzzy Logic Controller for Active Suspension using Half Car Model, International Conference on Control Automation Robotics \& Vision, Pg 2415-2420.

5. Fuller C R and Von Flotow A H. (1995). Active Control of Sound and Vibration, IEEE Control Systems, Vol 15, Issue 6.

6. He Rongbo and Zheng Shijie. (2013). Independent modal variable structure fuzzy active vibration control of thin plates laminated with photostrictive actuators, Chinese Journal of Aeronautics, Vol. 26, Issue 2, pg. 350-356.

7. Jae-Hung Han, Keun-Ho Rew and In Lee. (1997). An experimental study of activevibration control of composite structures with a piezo-ceramic actuator and a piezo-film sensor, Smart Material Structures, Volume 6, Number 5, Pg 549-558.

8. Venkateswarlu, G., Jhansi, C., \& Raju, P. Fuzzy Logic Based Maximum Power Point Tracking Technique For Partially Shaded Photovoltaic System.

9. Jean-Paul Henderson, Andrew Plummer and Nigel Johnston. (2018). An electro-hydrostatic actuator for hybrid active-passive vibration isolation, International Journal of Hydromechatronics, Vol. 1, No. 1.

10. Labane Chrif, Zemalache Meguenni Kadda. (2014). Aircraft Control System Using LQG and LQR Controller with Optimal Estimation - Kalman Filter Design, International Symposium on Aircraft Airworthiness, pg. 245 - 257.

11. Leventhall H G and L. Wong. (1998). A Review of Active Attenuation and Development of an Active Attenuator Open Refuge, HSE Contract Research Report No. 4/1988, W S Atkins Engineering Sciences, Surrrey, UK.

12. Mead J. D. (1999). Passive Vibration Control, John Wiley \& Sons.

13. Preumont A. (2001). Active Vibration Control, UniversiteLibre de Bruxelles, Brussels, Belgium.

14. Rahman N, M. N. Alam and M. Junaid. (2018). Active vibration control of composite shallow shells: An integrated approach, Journal of Mechanical Engineering and Sciences, Volume 12, Issue 1, pp. 3354-3369. 
15. Satyam Paul, Wen Yu and Xiaoou Li. (2018). Bidirectional active control of structures with type-2 fuzzy PD and PID, International Journal of Systems, Volume 49, Issue 4.

16. Sen M. Kuo and Dennis R. Morgan. (1999). Active Noise Control: A Tutorial Review, Proceedings of the IEEE, Vol. 87 , No. 6.

17. Tahseen Hashim Saleh. (2014). Active Vibration Control of a smart beam using LQR, PID \& Fuzzy logic Controllers, International Journal of Engineering and Technology, Volume 5, N0.2. 